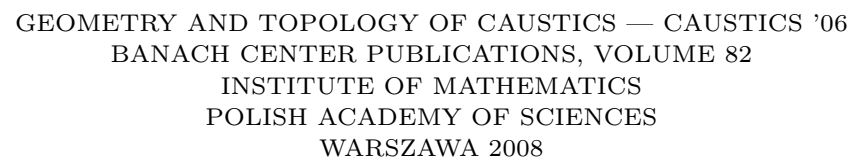

\title{
SINGULARITIES IN DRAWINGS OF SINGULAR SURFACES
}

\author{
ALAIN JOETS \\ Laboratoire de Physique des Solides, bât. 510 \\ Université de Paris-Sud, CNRS, UMR 8502, F-91405 Orsay Cedex, France \\ E-mail: joets@lps.u-psud.fr
}

\begin{abstract}
When drawing regular surfaces, one creates a concrete and visual example of a projection between two spaces of dimension 2. The singularities of the projection define the apparent contour of the surface. As a result there are two types of generic singularities: fold and cusp (Whitney singularities). The case of singular surfaces is much more complex. A priori, it is expected that new singularities may appear, resulting from the "interaction" between the singularities of the surface and the singularities of the projection. The problem has already been solved for the projection of a surface with a boundary. We consider here additional examples: the drawing of caustics and the drawing of the eversion of a sphere.
\end{abstract}

1. Introduction. The singularity theory created by H. Whitney has a direct application in the problem of representing a mathematical surface $M$. Indeed, drawing a regular surface makes a projection $f$ between $M$ and the plane of the drawing $P$. As every (differentiable) map between two spaces of dimension $2, f$ shows, generically, Whitney singularities, i.e. folds and cusps, where the rank of $f$ is less than 2 , its maximal possible value [Whitney]. In the case of drawings, it is common to call the image $A$ of the singular set $\Sigma$ of $f, A=f(\Sigma)$, the apparent contour. The apparent contour is the "local boundary" of the representation of $M$ in $P$. In addition to the cusp-points, the apparent contour may present special points called crossing points, where the apparent contour intersects itself. The crossing points are not related to the differential properties of $f$, but are simply points of coincidence.

If the surface $M$ is not regular but has itself singularities, the problem becomes much more complex. It must be analyzed on a case by case basis. To our knowledge, only a few cases have been studied. J. W. Bruce, P. J. Giblin and V. Goryunov succeeded in giving the list of the singularities (and of their metamorphoses) associated with the representation of a surface with a boundary [BruceGiblin, Goryunov]. Another result

2000 Mathematics Subject Classification: Primary 57R45; Secondary 57R42.

Key words and phrases: singularities of projections, caustics, sphere eversion.

The paper is in final form and no version of it will be published elsewhere. 


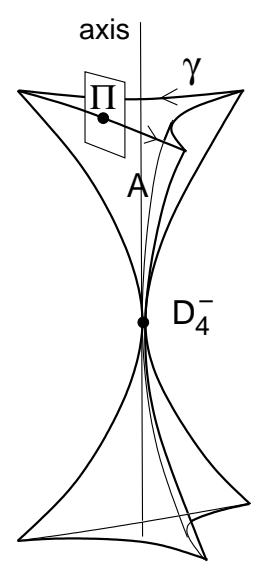

a)

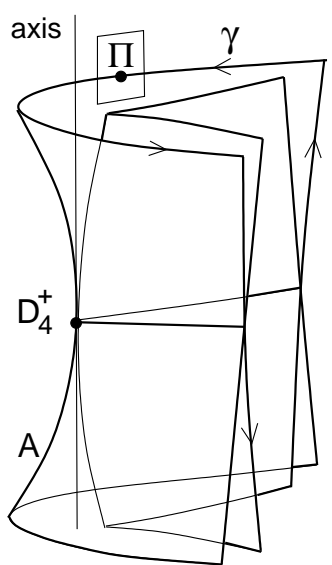

b)

Fig. 1. Apparent contour $A$ in the neighborhood a) of an elliptic umbilic $D_{4}^{-}$and b) of a hyperbolic umbilic $D_{4}^{+}$; the tangent plane $\Pi$ rotates around the axis by $\pi$ along the circuit $\gamma$.

concerns the representation of a surface with a corner [Babiker]. However this problem is of codimension 2, and does not appear naturally in simple problems.

The appearance of singularities in drawings of singular surfaces constitutes a natural problem every time that a new class of singular surfaces is discovered. For instance, an interesting and novel example is provided by the drawing of caustics in the $3 \mathrm{D}$ space (Lagrangian singularities). We shall present here a generic singularity in the drawings of caustics (a special contact point).

Another case is that of immersed surfaces. The knowledge of the rules governing the drawings of the surfaces is perhaps not necessary when only small parts are represented. However, it becomes essential for global configurations or in the case of surfaces depending on one parameter. We shall illustrate this with the example of the eversion of the sphere.

We wish to point out that, even if the computer graphics is useful to improve (through shadings, cuttings, etc) the visual representation of surfaces, it is not sufficient to give by itself a complete understanding of the drawing. This understanding necessitates above all the knowledge of the apparent contour, of its singularities and of its metamorphoses. In a sense, besides the computer vision, there exists a more fundamental vision that we might call singularity vision, i.e. vision based on the recognition of the singularities produced by the projection into the plane of the drawing. Thus, the singularity vision must precede the computer vision.

The aim of our paper is to illustrate the problem of drawing singular surfaces by two examples: the caustics and the eversion of the sphere. Our physicist's point of view, being rather practical and pragmatic, is in the spirit of G. Francis "Picturebook" [Francis]. Our work should be supplemented by a rigorous analysis and we hope that our results will stimulate new mathematical interest. 
2. Caustics in the physical space. Caustics in the physical space are associated with the singularities of a Lagrangian projection $\pi: L \rightarrow R^{3}$ between a 3D-Lagrangian space $L$, representing a congruence of rays, and our physical space $R^{3}=\{x, y, z\}$ [ArnGusVar]. The caustic $M$ is the image of the singular set $\Sigma \subset L$ of $\pi$, the set of points where the rank of $\pi$ is strictly less than 3 , its maximal possible value. $M=\pi(\Sigma)$ is a surface with singularities. Its regular part is formed by the fold-surface $A_{2}$. Its singular part is composed of cuspidal edges $A_{3}$ and of 3 types of point singularities: the swallowtails $A_{4}$, the elliptic umbilics $D_{4}^{-}$and the hyperbolic umbilics $D_{4}^{+} . M$ may also present 3 types of self-intersections: self-intersection lines $A_{2} A_{2}$, intersections between 2 parts $A_{2}$; triple points $T$, intersections between 3 parts $A_{2}$; and the points $A_{2} A_{3}$, intersections between a cusp-line $A_{3}$ and the surface $A_{2}$.

Projecting $M$ into the plane $P$ of the paper produces an apparent contour $A$. The generic point of $A$ is the fold point of the representation $f: M \rightarrow P$. The fold-lines $F$ possibly end at cusp-points $C$. Generically, the apparent contour does not pass through the point singularities $A_{4}, A_{2} A_{3}$, or $T$. However, it passes necessarily through the umbilics $D_{4}$. Indeed, since an umbilic $D_{4}$ has a corank equal to 2 , an axis is defined at the umbilic point. Is is not difficult to see that the tangent plane $\Pi$ to $M$ along a small circuit $\gamma$ around the umbilic, rotates by $\pi$ (Fig. 1). Consequently, there exists one point of $\gamma$ that contains the visual ray. The caustic being composed of 2 sheets, the apparent contour passes through the points $D_{4}$.

It is known that the contact of the representation of a line lying on a regular surface with the apparent contour $A$ is generically an ordinary contact (also called 2-fold contact [BruceGiblinCS]). In the case of a cusp-line, the situation is different: a cusp-line $A_{3}$ has, in the drawing, a 3-fold contact with the apparent contour. In other words, the drawing of a cusp-line passes tangentially through the apparent contour (Fig. 2). Inside the interior space of the cusp, the cusp-line is visible only at these 3 -fold contact points.

This special contact may be understood in the following way. We consider a congruence of normals (the rays) to a surface $\mathcal{W}$ (the initial wave front) given by its Monge form:

$$
\begin{aligned}
z= & \alpha+\frac{1}{2}\left(\beta_{x x} x^{2}+\beta_{y y} y^{2}\right)+\frac{1}{6}\left(\gamma_{x x x} x^{3}+3 \gamma_{x x y} x^{2} y+3 \gamma_{x y y} x y^{2}+\gamma_{y y y} y^{3}\right) \\
& +\frac{1}{24}\left(\delta_{x x x x} x^{4}+4 \delta_{x x x y} x^{3} y+6 \delta_{x x y y} x^{2} y^{2}+4 \delta_{x y y y} x y^{3}+\delta_{y y y y} y^{4}\right)+\ldots
\end{aligned}
$$

The axes $x$ and $y$ are taken along the principal directions. The current point $N=(X, Y, Z)$ along a normal to $\mathcal{W}$ is parametrised by 3 coordinates: $x$ and $y$ defining the normal passing through the point $(x, y, z(x, y))$ of $\mathcal{W}$ and a coordinate $s$ along the normal. The Lagrangian manifold $L$ is then parametrised by $x, y$ and $s$, and the Lagrangian projection $\pi$ is defined by $\pi(x, y, s)=N$. The singular set $\Sigma \subset L$ of the application $\pi$ is defined by $\operatorname{rk}(d \pi(x, y, s))<3$, that is to say by $\operatorname{det} \partial(X, Y, Z) / \partial(x, y, s)=0$. This equation is of second degree in $s: a(x, y) s^{2}+b(x, y) s+c(x, y)=0$. Each solution $s_{ \pm}(x, y)$ describes one of the two sheets $M_{ \pm}$of the caustic $\pi(\Sigma)$. Let us consider the sheet $M_{-}$. The current point $Q=(U, V, W)$ on $M_{-}$is parametrised by $x$ and $y: U(x, y)=X\left(x, y, s_{-}(x, y)\right)$, etc.

We now impose some constraints in order to analyse the contact of the cusp-line with the apparent contour. At first, the constant term $\alpha$ in the Monge form is chosen equal 


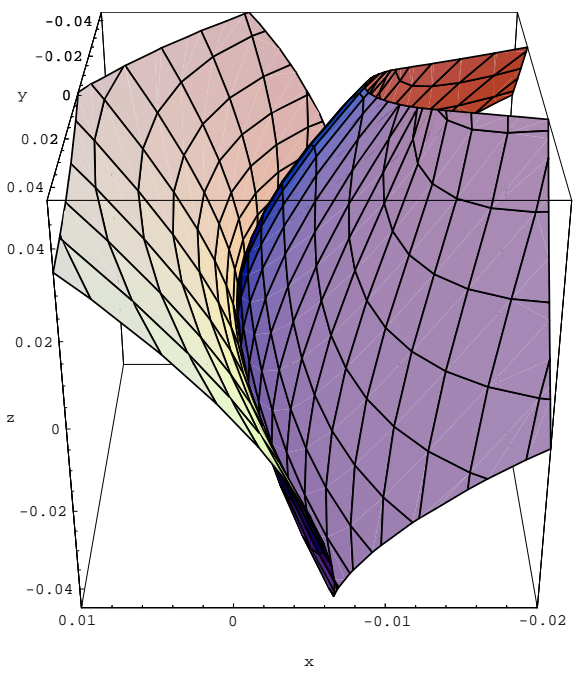

Fig. 2. Drawing of a cusp line $A_{3}$. The posterior part of the cusp-line is hidden by the right fold and the anterior part is hidden by the left fold, showing that the cusp-line represented passes through the apparent contour. The values of the parameters are: $\beta_{x x}=1.93, \beta_{y y}=\gamma_{x x y}=$ $\gamma_{y y y}=\delta_{x x x x}=\delta_{x x y y}=\delta_{x y y y}=\delta_{y y y y}=1, \gamma_{x y y}=3.5$.

to $-1 / \beta_{x x}\left(\beta_{x x}>\beta_{y y}\right)$ in order to make $M_{-}$passing through the origin: $Q(0,0)=0$. The cusp-line is defined as the Thom-Boardmann class $\Sigma^{1,1}$ [ArnGusVar], that is to say by the cancellation of 3 determinants $d_{i}: d_{1}=\operatorname{det} \partial(U, V) / \partial(x, y)$, etc. The expression of the $d_{i}$ allows one to see that the origin is a singularity $A_{3}$ if $\gamma_{x x x}=0$. Then, developing the $d_{i}$ to the second order in $x$ and $y$, we may also impose the tangent to $A_{3}$ at 0 to lie in the vertical plane $\{y, z\}$, if $\delta_{x x x y}=-3 \gamma_{x x y} \gamma_{x y y} /\left(\beta_{x x}-\beta_{y y}\right)$.

The tangent plane to $M_{-}$at the origin being the vertical plane $\{y, z\}$, we consider an orthogonal projection onto a plane $P$ perpendicular to $\{y, z\}$. The plane $P$ is defined by its normal $\vec{n}=(0, \sin a, \cos a)$. The projection $p$ is then given by $p(\vec{r})=\vec{r}-(\vec{n} \cdot \vec{r}) \vec{n}$, where $\vec{r}=(x, y, z)$. Let $u_{1}, u_{2}$ be the coordinates in $P$, the $x$ axis being projected on the $u_{1}$ axis and the $y$ and $z$ axes being projected on the $u_{2}$ axis. The apparent contour $A$ corresponds to the singularities of the map $f:(x, y) \mapsto p(Q(x, y))$. With our constraints, $A$ is a line passing through the origin and tangent the $u_{2}$-axis. We calculate that it is given by $u_{1}=k_{A} u_{2}^{2}+\ldots$, with

$$
k_{A}=-\frac{\beta_{x x}^{3} \gamma_{x y y}}{2\left(\gamma_{x x y} \cos a+\beta_{x x}\left(\beta_{x x}-\beta_{y y}\right) \sin a\right)^{2}}
$$

On the other hand, one calculates that the projection of the $A_{3}$-line in $P$ is also given by $u_{1}=k_{A} u_{2}^{2}+\ldots$, showing that this line and $A$ have a 3 -fold contact point (we have checked that $k_{A}$ is independent of the terms $\epsilon$ of higher order in the Monge form).

3. Immersed surfaces. The eversion of the sphere being realized by a family of immersions (differentiable applications of maximal rank), we have to consider first the representation of immersed surfaces, that is to say images of immersions $S^{2} \rightarrow R^{3}$ [MorinPetit]. 
3.1. Singularities of an immersed surface. An immersed surface may possess 2 types of special points: double points, forming self-intersection lines $I$ and triple points $T$ (Fig. 3 ).

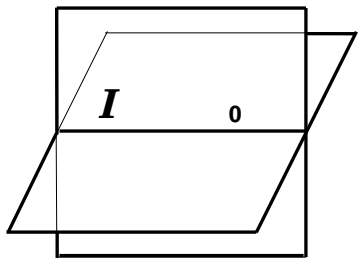

a)

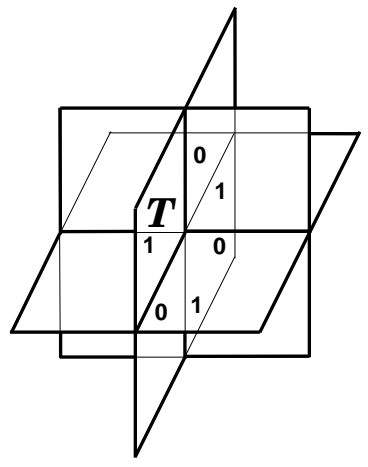

b)

Fig. 3. An immersed surface may have a) self-intersection lines $I$ and b) triple points $T$. The numbers stand for the screen numbers of the lines.

When we represent a self-intersection line, or any type of line, in the plane $P$ of the drawing, it is interesting to indicate the number of sheets encountered by our visual ray, between the line and our eye. Let us call this number the screen number of the line, which expresses the degree of non-visibility of the line. A line with a screen number 0 is visible. In $P$, a triple point $T$ appears as the intersection of 3 self-intersection lines. It is important to note that the screen number of each self-intersection line varies by 1 unit at $T$ (Fig. 3b).

There exist also in $P$ crossing points $I-I$ between two self-intersections lines (their screen number must differ from (at least) 2).

3.2. Apparent contour of an immersed surface. The generic point of the apparent contour $A$ of an immersed surface is the fold point. In the plane of the representation, a foldline bounds locally two sides: the side corresponding to the surface $M$, and the side external to $M$. It is useful to know the side (in $P$ ) corresponding to the surface (the "co-orientation" of the fold-line). In our drawings, this side is indicated by a very small arc of circle.

As in the case of regular surfaces, the apparent contour may have cusp-points $C$. The co-orientation in the neighborhood of $C$ points towards the interior of the cusp (see Fig. 4a). At the cusp-point $C$, the screen number of the fold-line changes by 1 unit.

In addition to the cusp-points, the apparent contour may have ordinary contact-points $\kappa$ with self-intersection lines $I$ (see Fig. $4 \mathrm{~b}$ ). At a point $\kappa$, the screen number of the foldline and that of the self-intersection line are exchanged. Of course, the co-orientation of $A$ points towards the line $I$.

The apparent contour $A$ may also have crossing points $F-I$ with the intersection-lines $I$, and self-intersection points $F-F$. 




a)



b)

Fig. 4. The apparent contour of an immersed surface may have a) cusp-points $C$, where the screen number of the fold-lines $F$ changes by one unit, b) contact-points $\kappa$ with a self-intersection line $I$, where the screen numbers of $F$ and $I$ are exchanged.

As noted in the preceding section, the apparent contour $A$, generically, does not pass through the triple points.

3.3. Metamorphoses of the apparent contour of a regular surface. In order to treat the eversion of the sphere, we need to know the metamorphoses of the apparent contour.
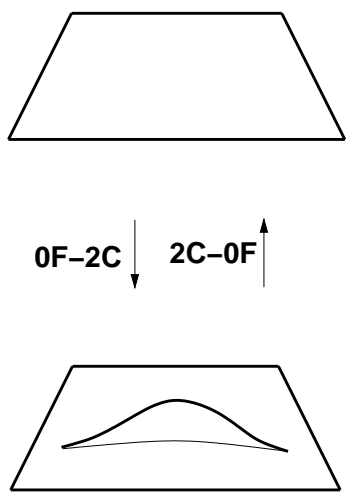

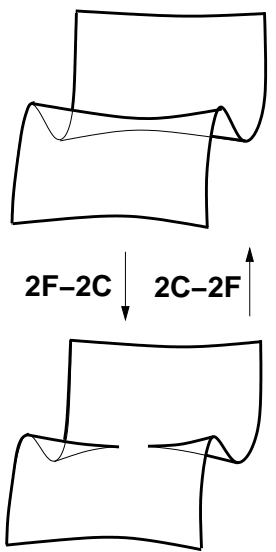

Fig. 5. The apparent contour of a regular surface may undergo 6 possible metamorphoses.

In the case of a regular surface, there exist 6 metamorphoses (see Fig. 5). In each case, two cusps are appearing or disappearing. The metamorphoses are distinguished by the fact that the cusps are appearing or disappearing and by the number of fold-lines $(0,1$, or 2 ) when the 2 cusps are absent. Consequently, we propose to designate them by a symbol containing this number of fold-lines ( $0 F$ for zero fold-line, etc). Moreover, the symbol $2 C$ is added at the end or at the beginning depending on the type of the metamorphosis: a creation or an annihilation of cusps. For instance, $0 F-2 C$ is the metamorphosis creating a pair of cusps starting from "nothing". $2 C-1 F$ is the metamorphosis annihilating a pair of cusps, the result being a single fold-line, etc. 
3.4. Metamorphoses of the apparent contour of an immersed surface. The metamorphoses of regular surfaces are also metamorphoses of immersed surfaces. In addition, there exist also metamorphoses typical of immersed surfaces. We give here the 4 metamorphoses appearing in the eversion of the sphere (see Fig. 6). We name them using the elements that they involve (the bifurcation parameter is the temporal parameter $\varepsilon$ ).

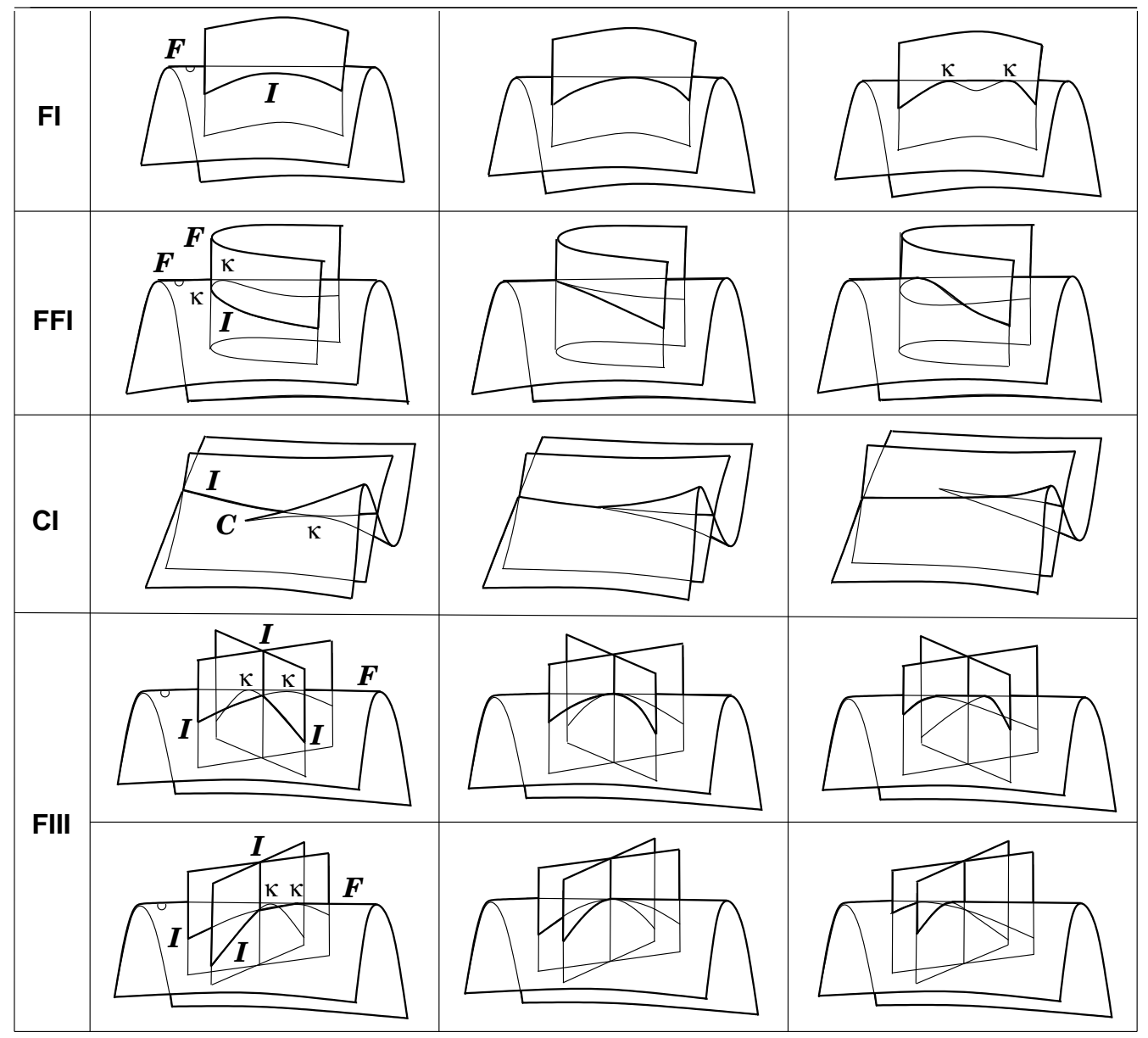

Fig. 6. 4 metamorphoses of the apparent contour of an immersed surface.

$F I$ : the metamorphosis $F I$ describes a self-intersection line $I$ coming into contact with a fold-line $F$ of the apparent contour. The line $I$ must be in the co-orientation of $F$. During the metamorphosis a pair of contact-points $\kappa$ is created $\left(F I^{+}\right)$or annihilated $\left(F I^{-}\right)$. An example of $F I$ may be provided by the following model: the fixed surface $z=y^{2}$ is cut by the moving surface $y=x^{2}-\varepsilon$. The projection is the orthogonal projection into the plane $\{x, z\}$. The metamorphosis occurs at $\varepsilon=0$. The distance between the two points $\kappa$ is of order $\sqrt{\varepsilon}$, whereas the domain limited by the fold and the intersection line has a thickness of order $\varepsilon^{2}$. 
FFI: the metamorphosis FFI involves two folded surfaces, intersecting along a line $I$. The line $I$ is in contact with each line $F$ by a contact-point $\kappa$. At the moment of the metamorphosis, the two $\kappa$ points are in coincidence and the line $I$ appears as a cuspidal curve. The metamorphosis creates or annihilates a self-intersection point of the line I. FFI may be represented by the following model: the fixed surface $z=(x+y)^{2}$ is cut by the moving surface $x=(y+\varepsilon)^{2}$.

$C I$ : the metamorphosis $C I$ involves a self-intersection line $I$ and a cusp-point $C$. The line $I$ has a contact-point $\kappa$ with one of the two fold-lines starting from $C$. During the metamorphosis, the point $\kappa$ glides along the fold-lines. It reaches $C$ at the moment of the bifurcation and then passes on the other fold-line. $C I$ may be represented by the following model: the fixed surface $z=y^{3}+x y$ is cut by the moving plane $y=\varepsilon$.

It is interesting to note that these 3 metamorphoses are in fact versions of singularities of projections of surfaces with a boundary [BruceGiblin, Goryunov]. More precisely, they correspond to the singularities of codimension 1 .

The metamorphosis FI corresponds to the semi-lips (and semi-beaks) of [BruceGiblin] and to the $A_{1}$ of [Goryunov]. The metamorphosis FFI corresponds to the boundary cusp of [BruceGiblin] and to the $B_{1}$ of [Goryunov]. The metamorphosis $C I$ corresponds to the semi-cusp of [BruceGiblin] and to the $3_{1}$ of [Goryunov]. In this comparison, the contact-point $\kappa$ corresponds to the semi-fold of [BruceGiblin]. In fact, the line $I$ of our 3 metamorphoses play exactly the role of the boundary of the surface considered in [BruceGiblin, Goryunov]. We now examine the fourth metamorphosis that involves 3 selfintersection lines, and, for that reason, has no connexion with the subject of singularities of projections of surfaces with a boundary:

$F I I I$ : the metamorphosis FIII involves a triple point, intersection point of 3 intersection lines $I$, and a fold-line $F$. During the metamorphosis, the triple point passes from the visible side of the fold to its invisible side. Simultaneously, a crossing-point between two lines $I$ passes through the triple point (in $P$ ). The metamorphosis has two variants; in one case the two points $\kappa$ are separated by the third intersection line; in the other case they lie on the same side. The metamorphosis is represented by the following model: the fixed surface $z=y^{2}$ is cut by the two moving surfaces $x=y-\varepsilon$ and $x= \pm 2(y-\varepsilon)$ (the sign is - for the first variant and + for the second variant). The distance of the triple point to $F$ is of order $\varepsilon^{2}$.

3.5. Crossings. In addition to the metamorphoses, there exist several types of modifications in which different lines $(I$ or $F$ ) come into contact and cross each other in the plane of projection $P$. For example two visible separate fold-lines may get closer, come into contact, and then cross, producing a pair of crossing points $F-F$. Between these two crossing points the screen number of one the two lines is changed by 2 units.

There are many types of crossings, involving 2 or 3 lines $I$ or $F$, but also points $\kappa, C, T$, etc. Some of them are analyzed in [Goryunov] and in [OhmotoAicardi]. The possibilities depend essentially on the screen number of the lines and on the co-orientation of the fold-lines. We do not give the details of these crossings, which are rather evident. 
4. Sphere eversion. The problem of the eversion of the sphere consists in giving an explicit way to turn a sphere inside out, a possibility proved theoretically by S. Smale in 1957 [Smale]. The eversion is realized by a 1-parameter family of "transversal immersions" undergoing, at some moments, certain transformations [MorinPetit]. Our purpose is not to give a new solution, but rather a new representation of the eversion of the sphere, based on the singularities of the apparent contour. We choose the solution exposed in [MorinPetit], which has the advantage to present two kinds of symmetries.

The first symmetry concerns the process everting the sphere: a first series of transformations is realized up to a "central model". Then, the central model is rotated by $\pi / 2$. The rotation exchanges the position of the inside of the sphere with that of its outside. Then, the opposite transformations are realized in the opposite order and the reversed sphere is thus obtained. The initial problem is reduced to that of the construction of the central model.

The second symmetry characterizes the surface itself: during the eversion, the surface possesses an axis of symmetry (binary axis). We choose this axis for the direction of the projection (this possibility is not exploited in [MorinPetit], which does not describe the apparent contours). Consequently our drawings will be symmetrical around a center.

In a sense, the two symmetries divide the number of bifurcations of the apparent contour by (roughly) 4 .

4.1. The 6 transformations. Six types of transformations are involved in the eversion of the sphere [MorinPetit] (Fig. 7).

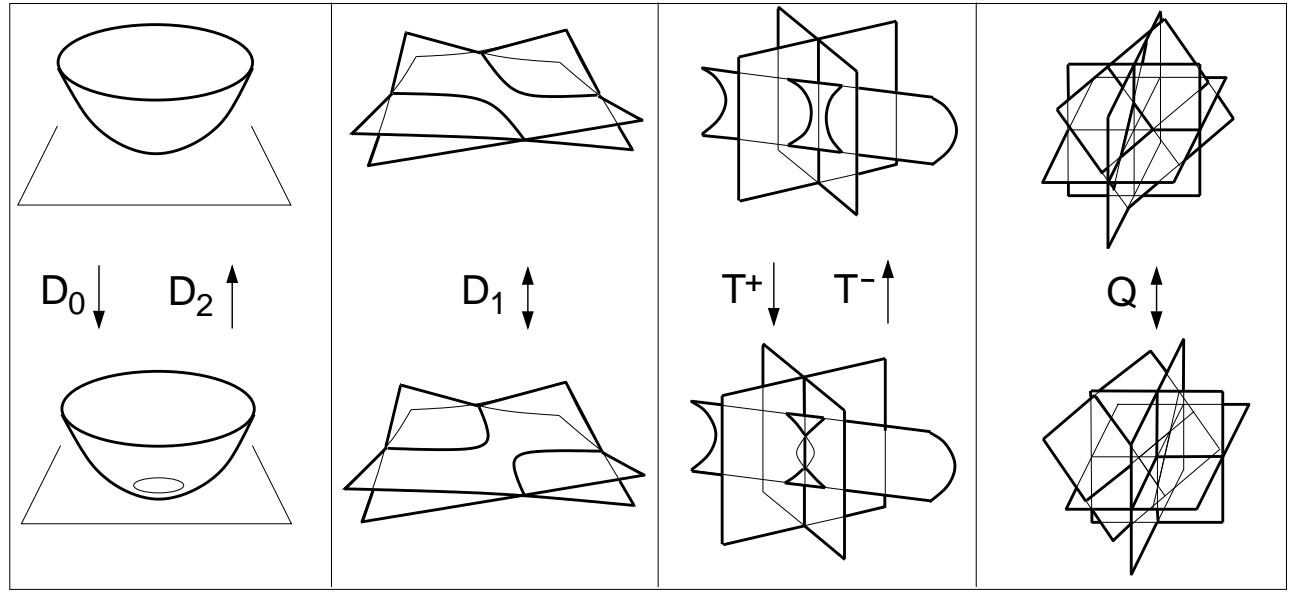

Fig. 7. The 6 transformations.

By the transformation $D_{0}$ a small self-intersection line $I$ forming a circle is created. A simple example of $D_{0}$ is provided by a sphere "crossing" a plane. Before the transformation the two surfaces are separate; at the moment of the transformation they have a contact at 1 point; after the transformation they intersect along a small circle.

The transformation $D_{2}$ is the transformation $D_{0}$ in the opposite sense: the small circle $I$ annihilates. 
The transformation $D_{1}$ is a deconnexion-reconnexion of two lines $I$.

By the transformation $T^{+}$a pair of triple points is created, resulting from the intersection of 3 surfaces.

The transformation $T^{-}$is the transformation $T^{+}$in the opposite sense: a pair of triple points annihilates.

The transformation $Q$ involves 4 surfaces, mutually intersecting. The intersection lines form a small tetrahedron, whose vertices are 4 triple points. During the transformation the size of the tetrahedron decreases; at the moment of the transformation it is reduced to 1 point; then it reappears after the transformation.

It is interesting to note that during these transformations, the apparent contour is (generically) not altered. We are now ready to see how the eversion of the sphere may be represented.

4.2. First step: creation of a first self-intersection line. We start with the standard sphere. Its apparent contour $A$ is a circle (Fig. 8). The sphere is "crushed" along the binary axis, and by the transformation $D_{0}$ the two poles cross, then forming a closed self-intersection line $I_{1}$. In this step, the apparent contour $A$ is not modified.

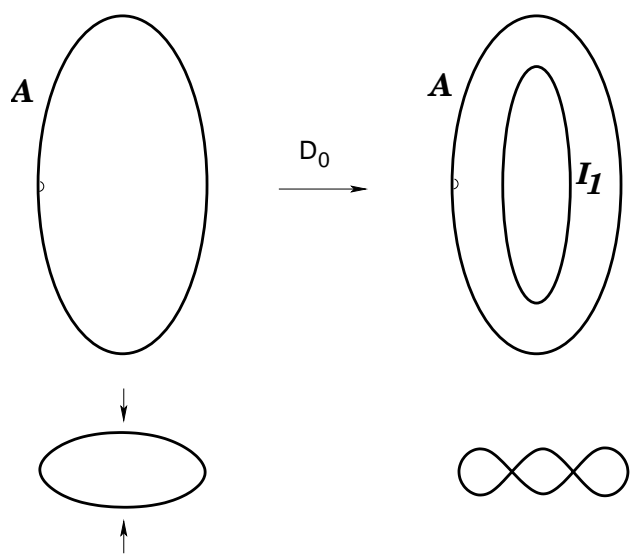

Fig. 8. First step: creation of a self-intersection line $I_{1}$ by the transformation $D_{0}$; the corresponding sections are indicated below.

4.3. Second step: creation of a second self-intersection line. The self-intersection line $I_{1}$ delimites 2 volumes: a lens-shaped volume at the center and a torus-shaped volume at the periphery. The second step consists in distorting the torus-shaped part until it intersects itself. The surface is first folded by a pair of metamorphoses $0 F-2 C$ (Fig. 9b). The apparent contour $A$ has now two new components. Then the folding increases and the internal parts of the two new components cross the line $I_{1}$ (Fig. 9c). The folding continues increasing and the external parts of the two new components come into contact with the line $I_{1}$. By two metamorphoses $F I$ four contact-points $\kappa$ appear (Fig. 9d). Finally, the two folds in the central part get closer, until they intersect themselves through a new transformation $D_{0}$ (Fig. 9e). A new self-intersection line, 8-shaped, $I_{2}$, is obtained. The line $I_{2}$ has 4 contact-points $\kappa$ with the apparent contour. 
The last 2 drawings of Fig. 9 show the deformations realized to make the surface more symmetrical: two crossings (Fig. 9f), followed by two metamorphoses FI (Fig. 9g).

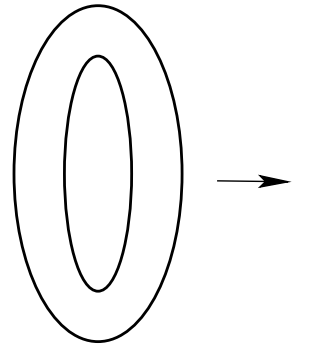

a)

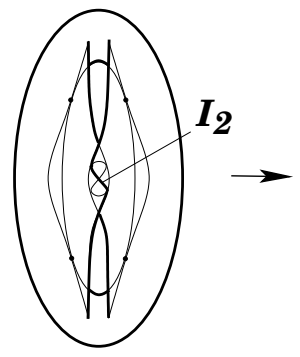

e)

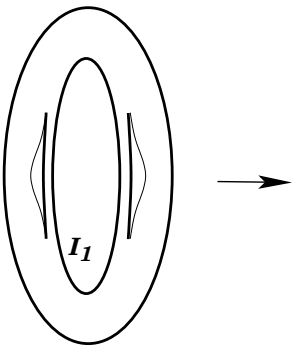

b)

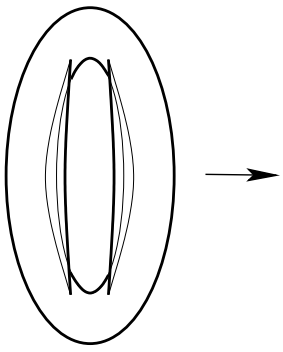

c)

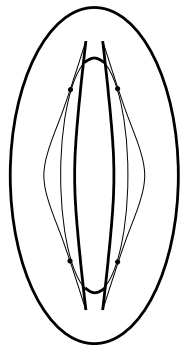

d)

Fig. 9. Second step: creation of a second self-intersection line $I_{2}$ by the transformation $D_{0}$ (between $d$ and $e$ ).

4.4. The problem of the symmetry. Due to the symmetry imposed, the second transformation $D_{0}$ has occurred on the apparent contour (Fig. 10a). This does not correspond to the generic case (see Fig. 7). Here, we want to understand how one may perturb slightly the transformation in order to recover the generic situation, that is to say the case without symmetry. Figure $10 \mathrm{~b}$ gives a solution.

a)



b)



Fig. 10. a) the transformation $D_{0}$ is seen along the axis of symmetry; b) the symmetry is broken and the final drawing is now obtained after the generic representation of $D_{0}$ plus 3 bifurcations of the apparent contour. 
The two fold-lines first cross. The two surfaces are now facing each other along the visual ray and the usual drawing of $D_{0}$ may be realized. The result is a small circle. The circle increases and comes in contact with the 2 folds, creating 2 pairs of contact-points $\kappa$. Finally the torsion of the circle is realized by a bifurcation FFI and the 8 is obtained.

4.5. Third step: the creation of 4 triple points. The third step affects the central 8 (Fig. 11). Because of the symmetry, we describe the modifications of only the left part of the 8 . They involve 3 self-intersection lines: the line $I_{1}$ and two parts of the line $I_{2}$ forming the 8 (Fig. 11a). In that position, a fold-line $F$ separates the two lines $I_{2}$ from the line $I_{1}$, and the transformation $T^{+}$is actually impossible. Consequently, a crossing is realized between $I_{1}$ and $F$ (Fig. 11b). The result is a self-intersection line of screen number 1 sandwiched between two self-intersection lines of screen number 0 . The transformation $T^{+}$may occur then, producing a pair of triple points (Fig. 11c). Of course, another transformation $T^{+}$ occurs symmetrically in the right part of the 8 (not shown on Fig. 11) and 4 triple points are in fact created simultaneously. The remaining metamorphoses make the surface more simple: a pair of contact-points is created by $F I^{+}$(Fig. 11d), a triple point moves to the other side of the fold by FIII (Fig. 11e), and finally two contact points annihilate by $F I^{-}$(Fig. 11f).

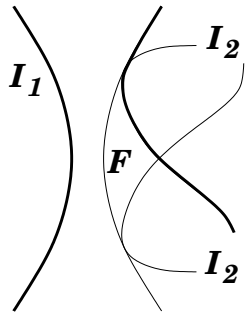

a)



d)

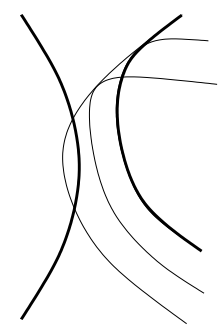

b)

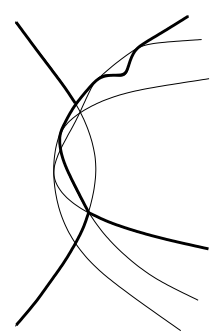

e)

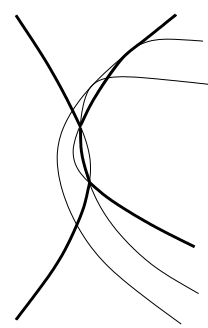

c)

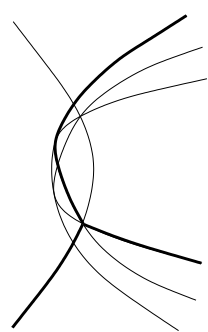

f)

Fig. 11. Third step: creation of 2 pairs of triple points by two transformations $T^{+}$(only the left central part is represented).

4.6. Central model. The remaining process is rather long and cumbersome. It involves the transformations $D_{1}$ and $Q$, the metamorphoses $F I, F F I, C I, 1 F-2 C, 2 C-2 F$, but also a great number of crossings. For that reason, we give up detailing the way leading to the central model. An explicit presentation shall be published elsewhere. Figure 12 shows the central model. It presents a higher symmetry: the axis of symmetry is now a 
4 -fold axis (invariance through a rotation of $\pi / 2$ ). However, if the internal and external sides of the sphere were represented by two different colors, the resulting figure would be invariant by a rotation of $\pi / 2$ followed by the exchange of the colors. This observation is at the root of the eversion of the sphere: the central model is rotated by $\pi / 2$, exchanging its internal and external sides, then all the transformations are realized in the opposite sense. The final result is the everted sphere.

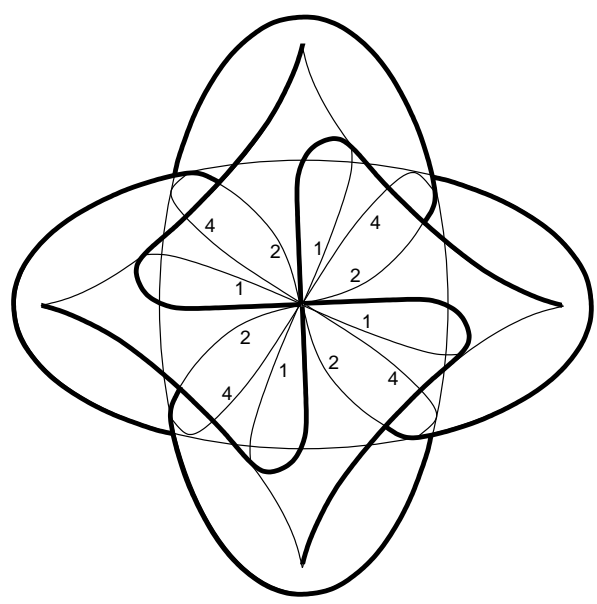

Fig. 12. a) The central model is invariant by a rotation of $\pi / 2$. The numbers indicate the screen numbers of the lines.

4.7. Cutting the surface. Using the representation of the surface $M$, one may deduce its section by any plane $V$ containing the direction of the projection. The trace of $V$ is a



a)

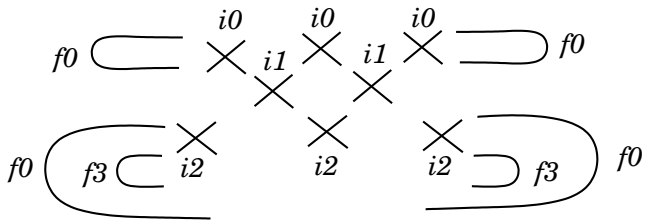

b)

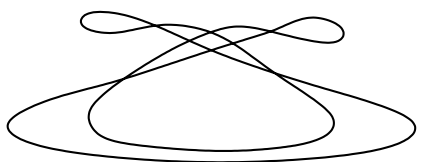

c)

Fig. 13. a) The representation of the distorted sphere $M$ before obtaining the central model; b) cutting the surface by a vertical plane $V$ (trace $v$ ), one obtains the series $f 0-f 0-f 3-i 2-$ $i 0-i 1-i 0 i 2-i 1-i 0-i 2-f 3-f 0-f 0 ; \mathrm{c})$ the section $M \cap V$ is drawn by connecting the arcs and the crosses. 
line $v$, cutting successively folds (points $f$ ) and the intersection-lines (points $i$ ). Noting the screen number of each point, we obtain a series of points denoted $f n$ and $\mathrm{im}$. Then, the series is represented in the plane $V$ : an arc of curve represents a point $f$ (the sense is given by the co-orientation of the fold-line) and a cross represents a point $i$. The arcs and the crosses are not positioned on the same line, but with a height depending on their screen number $n$ and $m$ respectively. Connecting these arcs and crosses, one obtains the section $M \cap V$. An example is given in Fig. 13 .

5. Conclusion. We have shown that the concrete process of drawing singular surfaces involves new types of singularities that result from the interaction between the singularities of the surface and the singularities of the projection into the plane of the drawing. Their understanding is necessary if we want to control complex graphical representations of singular surfaces, for instance those of the eversion of the sphere. We have also shown how to obtain sections of the surfaces.

Our results provide new elements for a "singularity vision", that is to say the recognition of the role of the singularities in the process of drawing and of seeing.

\section{References}

[ArnGusVar] V. I. Arnold, S. M. Gusein-Zade and A. N. Varchenko, Singularities of Differentiable Maps, Volume I, Birkhäuser, 1985.

[Babiker] H. Babiker, On the projection of surfaces with singular boundary, in: Singularities and Symplectic Geometry - Part VI, Singularity theory seminar, S. Janeczko (ed.), Warszawa, vol. 8 (2003), 1-17.

[BruceGiblin] J. W. Bruce and P. J. Giblin, Projections of surfaces with boundary, Proc. London Math. Soc. 60 (1990), 392-416.

[BruceGiblinCS] J. W. Bruce and P. J. Giblin, Curves and Singularities, Cambridge University Press, 1984.

[Francis] G. K. Francis, A Topological Picturebook, Springer-Verlag, New York, 1987.

[Goryunov] V. V. Goryunov, Projections of generic surfaces with boundaries, in: The Theory of Singularities and its Applications, V. I. Arnold (ed.), Advances in Soviet Mathematics 1, AMS, Providence RI, 1990, 157-200.

[MorinPetit] B. Morin and J.-P. Petit, Le retournement de la sphère, Pour La Science 15 (1979), 34-49.

[OhmotoAicardi] T. Ohmoto and F. Aicardi, First order local invariants of apparent contours, Topology 45 (2005), 27-45.

[Smale] S. Smale, A classification of immersions of the two-sphere, Transactions of the American Mathematical Society 90 (1958), 281-290.

[Whitney] H. Whitney, On singularities of mappings of Euclidean spaces, I, Ann. Math. 62 (1955), 374-410. 